\title{
Synchronization Assessment in Power Networks and Coupled Oscillators
}

\author{
Florian Dörfler, Michael Chertkov, and Francesco Bullo
}

\begin{abstract}
Synchronization problems in complex networks have recently attracted tremendous scientific interested. Moreover, assessing the existence, stability, and robustness of synchronous states is a pervasive topic in the operation of power networks. Scientists in both communities have long aimed to identify sharp synchronization conditions as functions of the network topology and parameters. This paper proposes an insightful approach to this problem based on algebraic graph theory. We present a novel synchronization condition applicable to a general coupled oscillator model. We rigorously establish that our condition is exact for various interesting network topologies and parameters. Via statistical studies we show that our condition predicts accurately the existence of stable synchronous solutions for generic networks as well as various power network test cases. From these statistical studies, we conclude that our proposed condition is correct for almost all network topologies and parameters. Indeed, we also show that there exist possibly-thin sets of network topologies and parameters, where our condition is not sufficiently tight.
\end{abstract}

\section{INTRODUCTION}

Synchronization is pervasive in the operation of power networks. All generating units of an interconnected grid must remain in strict synchronism while continuously following demand and rejecting disturbances. In face of the rising complexity of the future power grid and the stochastic disturbances caused by renewables, a deeper understanding of the system complexity becomes increasingly important.

A central question in the operation of a power network is "under which conditions on the network parameters and topology and the current load and generation profile, does there exist an optimal [1], stable [2]-[4], and robust [5]-[8] synchronous operating point". A more general concern is wether the load flow admits any solution [9], [10], or wether an existing solution vanishes in a saddle node bifurcation [11], [12]. All these questions are intimately intertwined, and various security indices have been proposed to quantify the robustness of an operating condition [13]. In general, accurate answers to the above questions are known only for radial (acyclic) distribution networks [1], [9], [13], only loose conditions are known for arbitrary networks [5], [7], [8], and various puzzling examples are known [6]. The assessment of an acceptable synchronous operating point and the quantification of its robustness will become more and more important in an increasingly complex and stressed grid.

F. Dörfler and F. Bullo are with the Center for Control, Dynamical Systems and Computation, University of California at Santa Barbara. M. Chertkov is with Theory Division \& Center for Nonlinear Studies at LANL and with New Mexico Consortium, Los Alamos, NM 87544. Email: \{dorfler,bullo\}@engineering.ucsb.edu, chertkov@lanl.gov. This material is based in part upon work supported by NSF grants IIS-0904501 and CPS-1135819. M. Chertkov acknowledges partial support of the DTRA Basic Research grant BRCALL08Per3D20022 via LANL and NSF award 1128437 via NMC.
Synchronization in complex networks recently attracted tremendous scientific interest [14]-[16]. Especially, the $\mathrm{Ku}-$ ramoto model of coupled oscillators [17] serves as prototypical example, and we refer to [18]-[21] for various applications and results. Despite the vast interest, the search for sharp and concise synchronization conditions for Kuramoto oscillators has been in vain so far. The available results to date include: exact synchronization conditions for specific network topologies [21]-[23], sufficient conditions for arbitrary topologies [19], [24], [25], and numerical investigations for random networks [26], [27]. Simulation studies indicate that the known sufficient conditions provide only loose estimates on the threshold from incoherence to synchrony. Literally, every review article on the Kuramoto model concludes by emphasizing the question for exact synchronization conditions for arbitrary networks [14]-[16], [18]-[21].

In summary, the power systems and the synchronization community ask for concise and sharp conditions that relate synchronization to the network topology and parameters [28].

Here, we present an insightful approach to synchronization. We consider a general coupled oscillator model that includes the Kuramoto model as well as various power network models [3], [4]. We study existence and local stability of synchronized solutions featuring bounded pair-wise angular distances, which are well-studied in synchronization [24], [25] and practically relevant in power systems [1]-[8], [11], [12]. Our analysis is based on algebraic graph theory and an equivalent reformulation of the synchronization problem which reveals the crucial role of cycles and cut-sets.

We propose a concise condition on the network topology and parameters, which includes the condition known for the homogeneous graph [21] and relaxes the conditions based on the algebraic connectivity [8], [14], [15], [19], [24]-[26] or the nodal degree [7], [24], [27], [29]. We prove that our condition is necessary and sufficient for the existence of a locally exponentially stable synchronization manifold for the sparsest (acyclic) and densest (homogeneous) network topologies, for the best (phase synchronizing) and the worst (cut-set inducing) network parameters, for cycles with symmetric parameters or of length strictly less than five, as well as combinations of these networks. Furthermore, we establish the statistical correctness of our condition for almost all topologies and parameters by extensive Monte Carlo simulations. As a negative result, we also show the existence of possibly-thin sets of networks for which our condition is not sufficiently tight. Finally, we validate the predictive power and high accuracy of our condition for the IEEE power system test cases 14, RTS 24, 30, New England 39, 57, RTS 96, 118, 300, and the Polish 2383 bus system. We envision the applicability of our condition as stability and robustness index.

The remainder of this section introduces some notation. 
Section II presents the considered models and our synchronization notions. Section III contains our analysis. Section IV illustrates our results through various test cases. Section $\mathrm{V}$ concludes the paper. The proofs of our theoretical results, the statistical results, and the numerical implementation is only sketched here, and we refer to [30] for details.

Geometry on $n$-torus: The set $\mathbb{S}^{1}$ denotes the unit circle, an angle is a point $\theta \in \mathbb{S}^{1}$, and an arc is a connected subset of $\mathbb{S}^{1}$. With slight abuse of notation, let $\left|\theta_{1}-\theta_{2}\right|$ denote the geodesic distance between two angles $\theta_{1}, \theta_{2} \in \mathbb{S}^{1}$. The $n$-torus $\mathbb{T}^{n}=\mathbb{S}^{1} \times \cdots \times \mathbb{S}^{1}$ is the direct sum of $n$ unit circles.

Vectors and functions: Given an $n$-tuple $\left(x_{1}, \ldots, x_{n}\right)$, let $x \in \mathbb{R}^{n}$ be the associated vector. Let $\mathbf{1}_{n}$ and $\mathbf{0}_{n}$ be the $n$ dimensional vectors of unit and zero entries, let $e_{n}^{i} \in \mathbb{R}^{n}$ be the $i$ th canonical unit vector, and define $\mathbf{1}_{n}^{\perp} \triangleq\left\{x \in \mathbb{R}^{n}\right.$ : $\left.x \perp \mathbf{1}_{n}\right\}$. For $x \in \mathbb{R}^{n}$, let $\sin (x)=\left(\sin \left(x_{1}\right), \ldots, \sin \left(x_{n}\right)\right)$ and $\arcsin (x)=\left(\arcsin \left(x_{1}\right), \ldots, \arcsin \left(x_{n}\right)\right)$, where the arcsin function is defined for the branch $[-\pi / 2, \pi / 2]$.

Algebraic graph theory: Given an undirected, connected, and weighted graph $G(\mathcal{V}, \mathcal{E}, A)$ induced by the symmetric and nonnegative adjacency matrix $A \in \mathbb{R}^{n \times n}$, we define the nodal degree by $\operatorname{deg}_{i}=\sum_{j=1}^{n} a_{i j}$. The Laplacian matrix $L \in \mathbb{R}^{n \times n}$ is defined by $L=\operatorname{diag}\left(\left\{\operatorname{deg}_{i}\right\}_{i=1}^{n}\right)-A$. If a number $\ell \in\{1, \ldots,|\mathcal{E}|\}$ and an arbitrary direction is assigned to each edge $\{i, j\} \in \mathcal{E}$, the oriented incidence matrix $B \in \mathbb{R}^{n \times|\mathcal{E}|}$ is defined component-wise as $B_{k \ell}=1$ if node $k$ is the sink node of edge $\ell$ and as $B_{k \ell}=-1$ if node $k$ is the source node of edge $\ell$; all other elements are zero. For $x \in$ $\mathbb{R}^{n}$, the vector $B^{T} x$ has components $x_{i}-x_{j}$ for any oriented edge from $j$ to $i$. If $\operatorname{diag}\left(\left\{a_{i j}\right\}_{\{i, j\} \in \mathcal{E}}\right)$ is the diagonal matrix of edge weights, then $L=B \operatorname{diag}\left(\left\{a_{i j}\right\}_{\{i, j\} \in \mathcal{E}}\right) B^{T}$. If the graph is connected, then $\operatorname{Ker}\left(B^{T}\right)=\operatorname{Ker}(L)=\operatorname{span}\left(\mathbf{1}_{n}\right)$, all $n-1$ remaining eigenvalues of $L$ are strictly positive (and real), and the second-smallest eigenvalue $\lambda_{2}(L)$ is called the algebraic connectivity. Since $L$ is singular, we will frequently use its Moore-Penrose inverse $L^{\dagger}$. The orthogonal vector spaces $\operatorname{Ker}(B)$ and $\operatorname{Ker}(B)^{\perp}=\operatorname{Im}\left(B^{T}\right)$ are denoted as cycle space and cut-set space, and they are spanned by vectors associated to cycles and cut-sets in the graph.

\section{Models And Synchronization Notions}

\section{A. General Coupled Oscillator Model}

Consider a weighted, connected, and undirected graph $G=(\mathcal{V}, \mathcal{E}, A)$ with $n$ nodes $\mathcal{V}$, partitioned node set $\mathcal{V}=$ $\mathcal{V}_{1} \cup \mathcal{V}_{2}$ and edge $\mathcal{E} \subset \mathcal{V} \times \mathcal{V}$ set induced by the adjacency matrix $A \in \mathbb{R}^{n \times n}$. Associated to this graph, consider the following model of $\left|\mathcal{V}_{1}\right| \geq 0$ second-order Newtonian and $\left|\mathcal{V}_{2}\right| \geq 0$ first-order kinematic phase oscillators

$$
\begin{aligned}
& M_{i} \ddot{\theta}_{i}+D_{i} \dot{\theta}_{i}=\omega_{i}-\sum_{j=1}^{n} a_{i j} \sin \left(\theta_{i}-\theta_{j}\right), \quad i \in \mathcal{V}_{1}, \\
& D_{i} \dot{\theta}_{i}=\omega_{i}-\sum_{j=1}^{n} a_{i j} \sin \left(\theta_{i}-\theta_{j}\right), \quad i \in \mathcal{V}_{2},
\end{aligned}
$$

where $\theta_{i} \in \mathbb{S}^{1}$ and $\dot{\theta}_{i} \in \mathbb{R}^{1}$ are the phase and frequency of oscillator $i \in \mathcal{V}, \omega_{i} \in \mathbb{R}^{1}$ and $D_{i}>0$ are the natural frequency and damping coefficient of oscillator $i \in \mathcal{V}$, and $M_{i}>0$ is inertial constant of oscillator $i \in \mathcal{V}_{1}$.

The dynamics (1) can be illustrated by points rotating around the unit circle with first or second-order dynamics, with respective natural frequencies $\omega_{i}$, and neighboring oscillators are coupled to another by Hookean springs with elasticity $a_{i j}$. The interesting dynamic behavior of the coupled oscillator model (1) arises from a competition between each oscillator's tendency to align with its natural frequency $\omega_{i}$ and the synchronization-enforcing coupling $a_{i j} \sin \left(\theta_{i}-\theta_{j}\right)$ with its neighbors. The coupled oscillator model (1) evolves on $\mathbb{T}^{n} \times \mathbb{R}^{\left|\mathcal{V}_{1}\right|}$, and features an important symmetry, namely the rotational invariance of the angular variable $\theta$.

Despite its simplicity, the coupled oscillator model (1) includes various models studied in the synchronization literature. For the parameters $\mathcal{V}_{1}=\emptyset$ and $D_{i}=1$ for all $i \in \mathcal{V}_{2}$, it reduces to the classic Kuramoto model [17]

$$
\dot{\theta}_{i}=\omega_{i}-\sum_{j=1}^{n} a_{i j} \sin \left(\theta_{i}-\theta_{j}\right), \quad i \in\{1, \ldots, n\} .
$$

Among other models listed in [21], the coupled oscillator model (1) also includes a variety of power network models.

\section{B. Structure-Preserving Power Network Model}

Consider a connected power network with generators $\mathcal{V}_{1}$ and load buses $\mathcal{V}_{2}$. The network is described by the symmetric nodal admittance matrix $Y \in \mathbb{C}^{n \times n}$ (augmented with the generator transient reactances). If the network is lossless and the voltage levels $\left|V_{i}\right|$ at all nodes $i \in \mathcal{V}_{1} \cup \mathcal{V}_{2}$ are constant, then the maximum real power transfer between any two nodes $i, j \in \mathcal{V}_{1} \cup \mathcal{V}_{2}$ is $a_{i j}=\left|V_{i}\right| \cdot\left|V_{j}\right| \cdot \Im\left(Y_{i j}\right)$. With this notation the swing dynamics of generator $i$ are given by

$$
M_{i} \ddot{\theta}_{i}+D_{i} \dot{\theta}_{i}=P_{\mathrm{m}, i}-\sum_{j=1}^{n} a_{i j} \sin \left(\theta_{i}-\theta_{j}\right), \quad i \in \mathcal{V}_{1},
$$

where $\theta_{i} \in \mathbb{S}^{1}$ and $\dot{\theta}_{i} \in \mathbb{R}^{1}$ are the generator rotor angle and frequency, $\theta_{j} \in \mathbb{S}^{1}$ for $j \in \mathcal{V}_{2}$ are the voltage phase angles at the load buses, and $P_{\mathrm{m}, i}>0, M_{i}>0$, and $D_{i}>0$ are the mechanical power input, inertia constant, and damping coefficient. We consider the following three load models.

1) Frequency-dependent loads: All buses are PV buses [4] and the real power drawn by load $i$ consists of a constant term $P_{1, i}>0$ and a frequency dependent term $D_{i} \dot{\theta}_{i}$ with $D_{i}>0$. The resulting in the real power balance equation is

$$
D_{i} \dot{\theta}_{i}+P_{1, i}=-\sum_{j=1}^{n} a_{i j} \sin \left(\theta_{i}-\theta_{j}\right), \quad i \in \mathcal{V}_{2} .
$$

The dynamics (3)-(4) are known as structure-preserving power network model [4], and equal the coupled oscillator model (1) for $\omega_{i}=P_{\mathrm{m}, i}, i \in \mathcal{V}_{1}$, and $\omega_{i}=-P_{1, i}, i \in \mathcal{V}_{2}$.

2) Constant power loads: If each load features a constant real power demand $P_{1, i}>0$, the load damping in (4) is neglected, that is, $D_{i}=0$ for $i \in \mathcal{V}_{2}$, and the angular distances $\left|\theta_{i}(t)-\theta_{j}(t)\right|<\pi / 2$ are bounded for each transmission line $\{i, j\} \in \mathcal{E}$ (this condition will be established in Section III), then the resulting differential-algebraic system has the same local stability properties as the dynamics (3)-(4), see [3].

3) Constant current loads: If each load demands a constant amount of current, then the linear current-balance equations are $I=Y V$, where $I \in \mathbb{C}^{n}$ and $V \in \mathbb{C}^{n}$ are the vectors of nodal current injections and voltages. After elimination of the bus variables $V_{i}, i \in \mathcal{V}_{2}$, through Kron reduction [29], the resulting dynamics assume the form (1) with $\mathcal{V}_{2}=\emptyset$. 


\section{Synchronization Notions and Conditions}

For $\gamma \in\left[0, \pi / 2\left[\right.\right.$, let $\bar{\Delta}_{G}(\gamma) \subset \mathbb{T}^{n}$ be the closed set of angle arrays $\left(\theta_{1}, \ldots, \theta_{n}\right)$ with the property $\left|\theta_{i}-\theta_{j}\right| \leq \gamma$ for $\{i, j\} \in \mathcal{E}$. Also, let $\Delta_{G}(\gamma)$ be the interior of $\bar{\Delta}_{G}(\gamma)$.

A solution $(\theta, \dot{\theta}): \mathbb{R}_{\geq 0} \rightarrow\left(\mathbb{T}^{n}, \mathbb{R}^{\left|\mathcal{V}_{1}\right|}\right)$ to the coupled oscillator model (1) is said to be synchronized if $\theta(0) \in \bar{\Delta}_{G}(\gamma)$ and there exists $\omega_{\text {sync }} \in \mathbb{R}^{1}$ such that $\theta(t)=\theta(0)+\omega_{\text {sync }} \mathbf{1}_{n} t$ $(\bmod 2 \pi)$ and $\dot{\theta}(t)=\omega_{\text {sync }} \mathbf{1}_{\left|\mathcal{V}_{1}\right|}$ for all $t \geq 0$. In other words, here, synchronized trajectories have the properties of frequency synchronization and phase cohesiveness, that is, all oscillators rotate with the same synchronization frequency $\omega_{\text {sync }}$ and all their phases belong to the set $\bar{\Delta}_{G}(\gamma)$.

For the coupled oscillator model (1), the explicit synchronization frequency is given by $\omega_{\text {sync }} \triangleq \sum_{i=1}^{n} \omega_{i} / \sum_{i=1}^{n} D_{i}$, see [21]. By transforming to a rotating frame with frequency $\omega_{\text {sync }}$ and by replacing $\omega_{i}$ by $\omega_{i}-D_{i} \omega_{\text {sync }}$, we obtain $\omega_{\text {sync }}=$ 0 (or equivalently $\omega \in \mathbf{1}_{n}^{\perp}$ ) corresponding to balanced power injections $P_{\mathrm{m}}=P_{1}$ in the model (3)-(4). Hence, without loss of generality, we assume that $\omega \in \mathbf{1}_{n}^{\perp}$ such that $\omega_{\text {sync }}=0$.

Given a point $r \in \mathbb{S}^{1}$ and an angle $s \in[0,2 \pi]$, let $\operatorname{rot}_{s}(r) \in \mathbb{S}^{1}$ be the rotation of $r$ counterclockwise by the angle $s$. For $\left(r_{1}, \ldots, r_{n}\right) \in \mathbb{T}^{n}$, define the equivalence class

$\left[\left(r_{1}, \ldots, r_{n}\right)\right]=\left\{\left(\operatorname{rot}_{s}\left(r_{1}\right), \ldots, \operatorname{rot}_{s}\left(r_{n}\right)\right) \in \mathbb{T}^{n} \mid s \in[0,2 \pi]\right\}$.

Clearly, if $\left(r_{1}, \ldots, r_{n}\right) \in \bar{\Delta}_{G}(\gamma)$, then $\left[\left(r_{1}, \ldots, r_{n}\right)\right] \subset$ $\bar{\Delta}_{G}(\gamma)$. Given $\theta \in \bar{\Delta}_{G}(\gamma)$, the set $\left([\theta], \mathbf{0}_{\left|\mathcal{V}_{1}\right|}\right) \subset \mathbb{T}^{n} \times \mathbb{R}^{\left|\mathcal{V}_{1}\right|}$ is a synchronization manifold of the coupled-oscillator model (1). A synchronized solution takes value in a synchronization manifold due to rotational symmetry. ${ }^{1}$

The coupled oscillator dynamics (1) feature (i) the synchronizing effect of the coupling described by the graph $G(\mathcal{V}, \mathcal{E}, A)$ and (ii) the de-synchronizing effect of the dissimilar natural frequencies $\omega \in \mathbf{1}_{n}^{\perp}$. Loosely speaking, synchronization occurs when the coupling dominates the dissimilarity. The coupling is typically quantified by the algebraic connectivity $\lambda_{2}(L)$ or the nodal degree $\operatorname{deg}_{i}$, and the dissimilarity is quantified by absolute norms $\|\omega\|_{p}$ or incremental norms $\left\|B^{T} \omega\right\|_{p}$, where $p \in\{2, \infty\}$. Sometimes, these conditions can be evaluated only numerically since they are statedependent [7], [8] or arise from a non-trivial linearization process (Master stability function) [14], [15]. In general, concise and accurate results are known only for specific topologies with uniform weights such as complete graphs [21] linear chains [22] and complete bipartite graphs [23]. For arbitrary graphs $G(\mathcal{V}, \mathcal{E}, A)$ and $\omega \in \mathbf{1}_{n}^{\perp}$, simulation studies indicate that the known conditions are conservative estimates on the threshold from incoherence to synchrony.

\section{SynCHRONIZATION ASSESSMENT}

\section{A. An Algebraic Approach to Synchronization Assessment}

It is known [21, Theorem 5.1 and 5.3] that existence and local exponential stability of synchronized solutions of the coupled oscillator model (1) can be entirely described by means of the first-order Kuramoto model (2).

\footnotetext{
${ }^{1}$ We restrict our attention to synchronized solutions satisfying $\left|\theta_{i}-\theta_{j}\right| \leq$ $\gamma<\pi / 2$ for $\{i, j\} \in \mathcal{E}$. Of course, there may exist other possible solutions but these are not practically relevant for power network applications due to thermal limits and stability constraints for the transmission lines.
}

Lemma 3.1: (Sync equivalence) Consider the coupled oscillator model (1) and the Kuramoto model (2). The following statements are equivalent for any $\gamma \in[0, \pi / 2[$ and any synchronization manifold $\left([\theta], \mathbf{0}_{\left|\mathcal{V}_{1}\right|}\right) \subset \bar{\Delta}_{G}(\gamma) \times \mathbb{R}^{\left|\mathcal{V}_{1}\right|}$.

(i) $[\theta]$ is a locally exponentially stable synchronization manifold the Kuramoto model (2); and

(ii) $\left([\theta], \mathbf{0}_{\left|\mathcal{V}_{1}\right|}\right)$ is a locally exponentially stable synchronization manifold of the coupled oscillator model (1).

Due to equivalence of local exponential stability, we will from now on focus on first-order Kuramoto model (2).

The following result is known in the synchronization literature [24], [25] as well as in power systems, where the saturation of a transmission line is connected to an eventual singularity of the load flow Jacobian [3]-[8], [11].

Lemma 3.2: (Stable equilibria in $\Delta_{G}(\pi / 2)$ ) Consider the Kuramoto model (2) with a connected graph $G(\mathcal{V}, \mathcal{E}, A)$, and let $\gamma \in[0, \pi / 2[$. The following statements hold:

1) The Jacobian of the Kuramoto model is given by $J(\theta)=-B \operatorname{diag}\left(\left\{a_{i j} \cos \left(\theta_{i}-\theta_{j}\right\}_{\{i, j\} \in \mathcal{E}) B^{T}}\right.\right.$

2) If there exists an equilibrium point $\theta^{*} \in \bar{\Delta}_{G}(\gamma)$, then it belongs to a locally exponentially stable equilibrium manifold $\left[\theta^{*}\right] \in \bar{\Delta}_{G}(\gamma)$; and

3) This equilibrium manifold is unique in $\bar{\Delta}_{G}(\gamma)$.

By Lemma 3.2, the problem of finding a locally stable synchronization manifold reduces to that of finding a fixed point $\theta^{*} \in \bar{\Delta}_{G}(\gamma)$ for some $\gamma \in[0, \pi / 2[$. The fixed-point equations of the Kuramoto model (2) read in vector form as

$$
\omega=B \operatorname{diag}\left(\left\{a_{i j}\right\}_{\{i, j\} \in \mathcal{E}}\right) \sin \left(B^{T} \theta\right) .
$$

Notice that $\omega$ necessarily has to be bounded such that fixed points of (5) exist: since $\sin (x) \in[-1,1]$, a necessary condition for the existence of a fixed point in $\bar{\Delta}_{G}(\gamma)$ is

$$
\operatorname{deg}_{i} \sin (\gamma)=\sum_{j=1}^{n} a_{i j} \sin (\gamma)>\left|\omega_{i}\right|, i \in\{1, \ldots, n\}
$$

In words, the nodal degree must be larger than the frequency dissimilarity (from the zero synchronization frequency).

In the following we aim to find conditions under which the fixed-point equation (5) admit a solution $\theta^{*} \in \bar{\Delta}_{G}(\gamma)$. We resort to a rather straightforward solution ansatz. By formally replacing each term $\sin \left(\theta_{i}-\theta_{j}\right)$ in the fixed-point equations (5) by a scalar $\psi_{i j}$, the fixed-point equation (5) can be equivalently written as the set of equations

$$
\begin{aligned}
& \omega=B \operatorname{diag}\left(\left\{a_{i j}\right\}_{\{i, j\} \in \mathcal{E}}\right) \psi, \\
& \psi=\sin \left(B^{T} \theta\right),
\end{aligned}
$$

where $\psi \in \mathbb{R}^{|\mathcal{E}|}$ is the vector with elements of $\psi_{i j}$. We will refer to equations (7) as the auxiliary-fixed point equations, and characterize their properties in the following theorem.

Theorem 3.3: (Properties of the fixed point equations) Consider the Kuramoto model (2) with graph $G(\mathcal{V}, \mathcal{E}, A)$ and $\omega \in \mathbf{1}_{n}^{\perp}$, its fixed-point equations (5), and the auxiliary fixed-point equations (7). The following statements hold:

1) Exact solution: Every solution of the auxiliary fixedpoint equations (7) is of the form

$$
\psi=B^{T} L^{\dagger} \omega+\psi_{\text {hom }},
$$


where the homogeneous solution $\psi_{\text {hom }} \in \mathbb{R}^{|\mathcal{E}|}$ satisfies $\operatorname{diag}\left(\left\{a_{i j}\right\}_{\{i, j\} \in \mathcal{E}}\right) \psi_{\text {hom }} \in \operatorname{Ker}(B)$; and

2) Exact synchronization condition: Let $\gamma \in[0, \pi / 2[$. The following statements are equivalent:

(i) There exists a locally exponentially stable solution $\theta^{*} \in \bar{\Delta}_{G}(\gamma)$ to the fixed-point equation (5).

(ii) There exists a solution $\theta \in \bar{\Delta}_{G}(\gamma)$ to

$$
B^{T} L^{\dagger} \omega+\psi_{\text {hom }}=\sin \left(B^{T} \theta\right) .
$$

for some $\psi_{\text {hom }} \in \operatorname{diag}\left(\left\{1 / a_{i j}\right\}_{\{i, j\} \in \mathcal{E}}\right) \operatorname{ker}(B)$.

(iii) There exists a solution $\psi \in \mathbb{R}^{|\mathcal{E}|}$ to the auxiliary fixed-point equations (7) of the form (9) satisfying the norm constraint $\|\psi\|_{\infty} \leq \sin (\gamma)$ and the cycle constraint $\arcsin (\psi) \in \operatorname{Im}\left(B^{T}\right)$.

If the equivalent statements (i), (ii), and (iii) are true, then we have the identities $B^{T} \theta^{*}=B^{T} \theta=\arcsin (\psi)$.

Note that the particular solution $B^{T} L^{\dagger} \omega$ to the auxiliary fixed-point equations (7) lives in the cut-set space $\operatorname{Ker}(B)^{\perp}$ and the homogenous solution $\psi_{\text {hom }}$ lives in the weighted cycle space $\psi_{\text {hom }} \in \operatorname{diag}\left(\left\{1 / a_{i j}\right\}_{\{i, j\} \in \mathcal{E}}\right) \operatorname{Ker}(B)$. As a consequence, for each cycle in the graph, we obtain one degree of freedom in choosing the homogeneous solution $\psi_{\text {hom }}$ as well as one nonlinear constraint $\arcsin (\psi) \in \operatorname{Im}\left(B^{T}\right)$.

Remark 1: (Comments on necessity) Notice that a minimum $\infty$-norm solution $\psi^{*}$ to equations (7) can be found via

$$
\min _{\psi \in \mathbb{R}^{|\mathcal{E}|}}\|\psi\|_{\infty} \quad \text { s.t } \quad \omega=B \operatorname{diag}\left(\left\{a_{i j}\right\}_{\{i, j\} \in \mathcal{E}}\right) \psi .
$$

By Theorem 3.3, such a minimum infinity-norm solution $\psi^{*}$ necessarily satisfies $\left\|\psi^{*}\right\|_{\infty} \leq \sin (\gamma)$ so that an equilibrium $\theta^{*} \in \bar{\Delta}_{G}(\gamma)$ exists. Hence, the condition $\left\|\psi^{*}\right\|_{\infty} \leq \sin (\gamma)$ is an optimal necessary synchronization condition.

\section{B. Synchronization Assessment for Specific Networks}

In this subsection we seek to establish that the condition

$$
\left\|B^{T} L^{\dagger} \omega\right\|_{\infty}<1
$$

is sufficient for the existence of locally exponentially stable equilibria in $\Delta_{G}(\pi / 2)$ or, more generally, that the condition

$$
\left\|B^{T} L^{\dagger} \omega\right\|_{\infty} \leq \sin (\gamma), \quad \gamma \in[0, \pi / 2[
$$

is sufficient for the existence of locally exponentially stable equilibria in $\bar{\Delta}_{G}(\gamma)$. Since the right-hand side of (12) is a concave function of $\gamma \in[0, \pi / 2[$ that achieves its supremum value at $\gamma^{*}=\pi / 2$, it follows that condition (12) implies (11).

Remark 2: (Interpretation of the sync condition) From a spectral viewpoint, condition (11) can be rewritten as

$$
\left\|B^{T} \cdot\left(U \operatorname{diag}\left(0,\left\{1 / \lambda_{i}\right\}_{i \in\{2, \ldots, n\}}\right)\right) \cdot\left(U^{T} \omega\right)\right\|_{\infty}<1,
$$

where $U \in \mathbb{R}^{n \times n}$ is the matrix of orthonormal eigenvectors of $L$ and $\lambda_{i}$ are the Laplacian eigenvalues. A sufficient condition for inequality (13) is the algebraic connectivity condition $\lambda_{2}(L)>\left\|B^{T} \omega\right\|_{\infty}$ similar to results developed in [8], [14], [15], [19], [24]-[26]. Likewise, a necessary condition for (13) is $2 \max _{i \in\{1, \ldots, n\}} \operatorname{deg}_{i} \geq \lambda_{n}(L)>2\|\omega\|_{\infty}$ resembling the necessary condition (6) and the conditions [7], [24], [27], [29]. Clearly, when compared to (13), both of the previous conditions feature only one of $n-1$ non-zero Laplacian eigenvalues and are overly conservative. Finally, condition (12) reduces to the synchronization condition for classic Kuramoto oscillators with homogeneous coupling [21].

In power systems, the equilibrium equations of (1), $\omega_{i}=$ $\sum_{j=1}^{n} a_{i j} \sin \left(\theta_{i}-\theta_{j}\right)$, are known as $A C$ power flow equations. By conventional engineering wisdom, the AC power flow equations can be well approximated by the so-called $D C$ power flow equations $\omega_{i}=\sum_{j=1}^{n} a_{i j}\left(\theta_{i}-\theta_{j}\right)$ for sufficiently small phase distances $\max _{\{i, j\} \in \mathcal{E}}\left|\theta_{i}-\theta_{j}\right| \leq \gamma \ll 1$. In vector form the DC power flow equations read as $\omega=L \theta$ and their solution $\theta^{*}$ has the phase differences $B^{T} \theta^{*}=B^{T} L^{\dagger} \omega$. According to condition (12), the worst-case phase distance $\left\|B^{T} L^{\dagger} \omega\right\|_{\infty}$ obtained by the DC power flow needs to be smaller than $\sin (\gamma)$, such that the solution to the AC power flow satisfies $\max _{\{i, j\} \in \mathcal{E}}\left|\theta_{i}-\theta_{j}\right| \leq \gamma$. Thus, condition (12) extends the common DC approximation from infinitesimally small angles $\gamma \ll 1$ to large angles $\gamma \in[0, \pi / 2[$.

The above linear interpretation is not only insightful but also practical since condition (12) can be quickly evaluated by numerically solving the sparse system $\omega=L \theta$.

The exact state-dependent synchronization conditions in Theorem 3.3 can be easily evaluated for the sparsest (acyclic) and densest (homogeneous) topologies and for "worst-case" (cut-set inducing) and "best" (identical) natural frequencies. For all of these cases the scalar condition (12) is sharp.

Theorem 3.4: (Sync condition for extremal graphs and parameters) Consider the Kuramoto model (2) with connected graph $G(\mathcal{V}, \mathcal{E}, A)$ and $\omega \in \mathbf{1}_{n}^{\perp}$ and the inequality condition (12) for $\gamma \in[0, \pi / 2[$. The following statements hold:

(G1) Exact synchronization condition for acyclic graphs: Assume that $G(\mathcal{V}, \mathcal{E}, A)$ is an acyclic graph. There exists an exponentially stable equilibrium $\theta^{*} \in \bar{\Delta}_{G}(\gamma)$ if and only if condition (12) holds. Moreover, in this case we have that $B^{T} \theta^{*}=\arcsin \left(B^{T} L^{\dagger} \omega\right) \in \bar{\Delta}_{G}(\gamma)$;

(G2) Tight synchronization condition for homogeneous graphs: Assume that $G(\mathcal{V}, \mathcal{E}, A)$ is a homogeneous graph, that is, there is $K>0$ such that $a_{i j}=K$ for all distinct $i, j \in\{1, \ldots, n\}$. Consider a compact interval $\Omega \subset \mathbb{R}$, and let $\Omega \subset \mathbb{R}^{n}$ be the set of vectors with components $\Omega_{i} \in \Omega$ for $i \in\{1, \ldots, n\}$. For all $\omega \in$ $\boldsymbol{\Omega}$ there is an exponentially stable equilibrium $\theta^{*} \in$ $\bar{\Delta}_{G}(\gamma)$ if and only if condition (12) holds;

(G3) Exact synchronization condition for cut-set inducing natural frequencies: Let $\Omega_{1}, \Omega_{2} \in \mathbb{R}$, and let $\Omega \subset \mathbb{R}^{n}$ be the set of bipolar vectors with components $\boldsymbol{\Omega}_{i} \in\left\{\Omega_{1}, \Omega_{2}\right\}$ for $i \in\{1, \ldots, n\}$. For all $\omega \in L \boldsymbol{\Omega}$ there exists an exponentially stable equilibrium $\theta^{*} \in$ $\bar{\Delta}_{G}(\gamma)$ if and only if condition (12) holds. Moreover, $\Omega$ induces a cut-set: if $\left|\Omega_{2}-\Omega_{1}\right|=\sin (\gamma)$, then for $\omega=L \Omega$ we obtain the exponentially stable equilibrium $\theta^{*} \in \bar{\Delta}_{G}(\gamma)$ satisfying $B^{T} \theta^{*}=\arcsin \left(B^{T} \Omega\right)$, that is, for all $\{i, j\} \in \mathcal{E},\left|\theta_{i}^{*}-\theta_{j}^{*}\right|=0$ if $\boldsymbol{\Omega}_{i}=\boldsymbol{\Omega}_{j}$ and $\left|\theta_{i}^{*}-\theta_{j}^{*}\right|=\gamma$ if $\boldsymbol{\Omega}_{i} \neq \boldsymbol{\Omega}_{j}$; and

(G4) Asymptotic correctness: In the limit $\omega \rightarrow \mathbf{0}_{n}$, there exists an exponentially stable equilibrium $\theta^{*} \in$ $\bar{\Delta}_{G}(\gamma)$ if and only if condition (12) holds. Moreover, $\lim _{\omega \rightarrow \mathbf{0}_{n}}\left(B^{T} \theta^{*}\right)_{i} / \arcsin \left(B^{T} L^{\dagger} \omega\right)_{i}=1, i \in\{1, \ldots,|\mathcal{E}|\}$. 
Theorem 3.3 shows that the solvability of the fixed-point equations (5) is inherently related to the cycle constraints. The following lemma establishes feasibility of a single cycle.

Lemma 3.5 (Single cycle feasibility): Consider the $\mathrm{Ku}$ ramoto model (2) with a cycle graph $G(\mathcal{V}, \mathcal{E}, A)$ and $\omega \in \mathbf{1}_{n}^{\perp}$. Without loss of generality, assume that the edges are labeled by $\{i, i+1\} \bmod n$ for $i \in\{1, \ldots, n\}$ and $\operatorname{Ker}(B)=$ $\operatorname{span}\left(\mathbf{1}_{n}\right)$. Define $x \in \mathbf{1}_{n}^{\perp}$ and $y \in \mathbb{R}_{>0}^{n}$ uniqely by $x \triangleq$ $B^{T} L^{\dagger} \omega$ and $y_{i} \triangleq a_{i,(i+1)} \bmod n>0$ for $i \in\{1, \ldots, n\}$. Let $\gamma \in[0, \pi / 2[$. The following statements are equivalent:

(i) There exists a stable equilibrium $\theta^{*} \in \bar{\Delta}_{G}(\gamma)$; and

(ii) the function $f:\left[\lambda_{\min }, \lambda_{\max }\right] \rightarrow \mathbb{R}$, with domain boundaries $\lambda_{\max }=\min _{i \in\{1, \ldots, n\}} \frac{\sin (\gamma)-x_{i}}{y_{i}}$ and $\lambda_{\min }=$ $\max _{i \in\{1, \ldots, n\}} \frac{-\sin (\gamma)-x_{i}}{y_{i}}$ and defined by $f(\lambda)=$ $\sum_{i=1}^{n} \arcsin \left(x_{i}+\lambda y_{i}\right)$, satisfies $f\left(\lambda_{\min }\right)<0<f\left(\lambda_{\max }\right)$. If both equivalent statements 1) and 2) are true, then $B^{T} \theta^{*}=$ $\arcsin \left(x+\lambda^{*} y\right)$, where $\lambda^{*} \in\left[\lambda_{\min }, \lambda_{\max }\right]$ satisfies $f\left(\lambda^{*}\right)=0$.

The condition in Lemma 3.5 leads to the following result.

Theorem 3.6: (Sync conditions for cycle graphs) Consider the Kuramoto model (2) with a cycle graph $G(\mathcal{V}, \mathcal{E}, A)$ and $\omega \in \mathbf{1}_{n}^{\perp}$ and the inequality condition (12) for $\gamma \in$ $[0, \pi / 2[$. The following statements hold.

(C1) Exact sync condition for symmetric natural frequencies: Assume that $\omega \in \mathbf{1}_{n}^{\perp}$ is such that $B^{T} L^{\dagger} \omega$ is a symmetric vector ${ }^{2}$. There is an exponentially stable equilibrium $\theta^{*} \in \bar{\Delta}_{G}(\gamma)$ if and only if condition (12) holds. Moreover, $B^{T} \theta^{*}=\arcsin \left(B^{T} L^{\dagger} \omega\right)$.

(C2) Tight sync condition for low-dimensional cycles: Assume the network contains $n \in\{3,4\}$ oscillators. Consider a compact interval $\Omega \subset \mathbb{R}$, and let $\Omega \in \mathbb{R}^{n}$ be the set of vectors with components $\Omega_{i} \in \Omega$ for $i \in\{1, \ldots, n\}$. For all $\omega \in L \Omega$ there exists an exponentially stable equilibrium $\theta^{*} \in \bar{\Delta}_{G}(\gamma)$ if and only if condition (12) holds.

(C3) General cycles and network parameters: In general for $n \geq 5$ oscillators, condition (11) does not guarantee $^{3}$ existence of an equilibrium $\theta^{*} \in \Delta_{G}(\pi / 2)$. As a sufficient condition, there exists an exponentially stable equilibrium $\theta^{*} \in \bar{\Delta}_{G}(\gamma), \gamma \in[0, \pi / 2[$ if

$$
\left\|B^{T} L^{\dagger} \omega\right\|_{\infty} \leq \frac{\min _{\{i, j\} \in \mathcal{E}} a_{i j} \cdot \sin (\gamma)}{\max _{\{i, j\} \in \mathcal{E}} a_{i j}+\min _{\{i, j\} \in \mathcal{E}} a_{i j}} .
$$

Let a patched network $\{G(\mathcal{V}, \mathcal{E}, A), \omega\}$ be a collection of subgraphs and natural frequencies $\omega \in \mathbf{1}_{n}^{\perp}$, where $(i)$ each subgraph is connected, (ii) in each subgraph one of the conditions (G1),(G2),(G3),(G4), (C1), or (C2) is satisfied, (iii) the subgraphs are connected to another through edges $\{i, j\} \in \mathcal{E}$ satisfying $\left\|\left(e_{|\mathcal{E}|}^{i}-e_{|\mathcal{E}|}^{j}\right)^{T} L^{\dagger} \omega\right\|_{\infty} \leq \sin (\gamma)$, and (iv) the set of cycles in the overall graph $G(\mathcal{V}, \mathcal{E}, A)$ is equal to the union of the cycles of all subgraphs. Since a patched graph satisfies the synchronization condition (12) as well the norm and cycle constraints, we can state the following result.

Corollary 3.7: (Sync condition for a patched network) Consider the Kuramoto model (2) with a patched network

\footnotetext{
${ }^{2}$ A vector $x \in \mathbf{1}_{n}^{\perp}$ is symmetric if its histogram is symmetric, i.e., up to permutation of its elements, $x$ is of the form $x=[-c,+c]^{T}$ for $n$ even and some $c \in \mathbb{R}^{n / 2}$ and $x=[-c, 0,+c]^{T}$ for $n$ odd and some $c \in \mathbb{R}^{(n-1) / 2}$.

${ }^{3} \mathrm{~A}$ carefully designed cyclic counterexample is reported in [30].
}

$\{G(\mathcal{V}, \mathcal{E}, A), \omega\}$, and let $\gamma \in[0, \pi / 2[$. There is an exponentially stable equilibrium $\theta^{*} \in \bar{\Delta}_{G}(\gamma)$ if condition (12) holds.

\section{Statistical Synchronization Assessment}

After having analytically established condition (12) for a variety of particular network topologies and parameters, we seek to establish its correctness for arbitrary networks. Extensive simulation studies lead us to the conclusions that the proposed condition (12) is correct in the generic case. In order to validate this hypothesis, we conducted Monte Carlo simulation studies over a wide range of natural frequencies $\omega_{i}$, network sizes $n$, coupling weights $a_{i j}$, and different random graph models of varying degrees of sparsity and randomness. For $1.2 \cdot 10^{6}$ samples of such nominal networks, we numerically solve equation (5) and test the hypothesis

$$
\mathcal{H}:\left\|B^{T} L^{\dagger} \omega\right\|_{\infty} \leq \sin (\gamma) \Longrightarrow \exists \theta^{*} \in \bar{\Delta}_{G}(\gamma) .
$$

The detailed Monte Carlo results can be found in the journal version of this paper [30]. By invoking the Chernoff bound, our simulations establish the following probabilistic result:

Statistical result: With at least $99 \%$ confidence and least $99 \%$ accuracy, the hypothesis $\mathcal{H}$ is true with $99.97 \%$ probability for a nominal network.

From this statistical result, we deduce that the proposed condition (12) is sufficient for almost all networks, and it fails for possibly-thin sets of topologies and parameters. In particular, for $0.03 \%$ of all cases, condition (12) is not sufficiently tight and fails marginally with an error of order $\mathcal{O}\left(10^{-4}\right)$.

Overall, our analytical results and statistical studies validate the correctness of the proposed condition (11). We refer to [30] for further investigations on the accuracy and the predictive power of condition (12) in random networks.

\section{SynC Assessment fOR POWER Networks}

We envision that condition (12) can be applied to quickly assess synchronization and robustness in power networks under volatile operating conditions. Since real-world power networks are carefully engineered systems with particular network topologies and parameters, we do not extrapolate the previous statistical results to power grids. Rather, we consider ten widely-established IEEE power network test cases.

Under nominal operating conditions, the power generation is optimized to meet the forecast demand, while obeying the $\mathrm{AC}$ power flow laws and respecting the thermal limits of each transmission line. In order to test the synchronization condition (12) in a volatile smart grid scenario, we make the following changes to the nominal network: 1) We assume fluctuating demand and randomize $50 \%$ of all loads to deviate from the forecasted loads with Gaussian statistics (with standard deviation 0.3 p.u.). 2) We assume that the grid is penetrated by renewables with severely fluctuating power outputs, and we randomize $33 \%$ of all generating units to deviate from the nominally scheduled generation with Gaussian statistics (with standard deviation 0.3 p.u.). 3) The fluctuations are mitigated by fast-ramping generation (or fast-response energy storage), and controllable loads. Here, we assume that the grid is equipped with $10 \%$ fast-ramping generation and $10 \%$ controllable loads, and the power imbalance (caused by fluctuating demand and generation) is 
TABLE I

\begin{tabular}{|l|l|l|l|}
\hline Randomized test case & Correctness & Accuracy & Cohesiveness \\
\hline \hline Chow 9 bus system & a lways true & $4.1218 \cdot 10^{-5}$ & 0.12889 \\
\hline IEEE 14 bus system & always true & $2.7995 \cdot 10^{-4}$ & 0.16622 \\
\hline IEEE RTS 24 & always true & $1.7089 \cdot 10^{-3}$ & 0.22309 \\
\hline IEEE 30 bus system & always true & $2.6140 \cdot 10^{-4}$ & 0.1643 \\
\hline New England 39 & always true & $6.6355 \cdot 10^{-5}$ & 0.16821 \\
\hline IEEE 57 bus system & always true & $2.0630 \cdot 10^{-2}$ & 0.20295 \\
\hline IEEE RTS 96 & always true & $2.6076 \cdot 10^{-3}$ & 0.24593 \\
\hline IEEE 118 bus system & always true & $5.9959 \cdot 10^{-4}$ & 0.23524 \\
\hline IEEE 300 bus system & always true & $5.2618 \cdot 10^{-4}$ & 0.43204 \\
\hline $\begin{array}{l}\text { Polish 2383 bus } \\
\text { system (winter 99) }\end{array}$ & always true & $4.2183 \cdot 10^{-3}$ & 0.25144 \\
\hline
\end{tabular}

uniformly dispatched among these adjustable sources. For each of the ten IEEE test cases, we construct 1000 random realizations of the scenario 1), 2), and 3) described above, we numerically check for the existence of a synchronous solution, and we compare the numerical solution with the results predicted by our synchronization condition (12).

Our findings are reported in Table I, and further details are provided in [30]. "Correctness" is evaluated according to the criterion $\left\|L^{\dagger} \omega\right\|_{\mathcal{E}, \infty} \leq \sin (\gamma) \Longrightarrow \max _{\{i, j\} \in \mathcal{E}} \mid \theta_{i}^{*}-$ $\theta_{j}^{*} \mid \leq \gamma$, "Accuracy" is evaluated by $\max _{\{i, j\} \in \mathcal{E}} \mid \theta_{i}^{*}-$ $\theta_{j}^{*} \mid-\arcsin \left(\left\|L^{\dagger} \omega\right\|_{\mathcal{E}, \infty}\right)$, and "Cohesiveness" is evaluated by $\max _{\{i, j\} \in \mathcal{E}}\left|\theta_{i}^{*}-\theta_{j}^{*}\right|$. The accuracy and cohesiveness results are averaged over all instances. It can be observed that condition (12) predicts the correct phase cohesiveness with extremely high accuracy even for large-scale networks.

\section{COnClusions}

We have presented an insightful approach to synchronization in coupled phase oscillators based on algebraic graph theory. We proposed a novel synchronization condition which is provably exact for various interesting network topologies and parameters. Furthermore, we showed through statistical studies that our condition appears is accurate for generic networks as well as various power system test cases.

Since there exist possibly-thin sets of topologies and parameters for which the proposed condition is not sufficiently tight, and further research must be carried out. Also, the results in this article are based on models requiring the classic separation of angle and voltage dynamics in active and reactive power flows. We are currently also working on extending the present results to non-constant voltages.

\section{REFERENCES}

[1] J. Lavaei, D. Tse, and B. Zhang, "Geometry of power flows in tree networks," in IEEE Power \& Energy Society General Meeting, San Diego, CA, USA, 2012, to appear.

[2] K. S. Chandrashekhar and D. J. Hill, "Cutset stability criterion for power systems using a structure-preserving model," International Journal of Electrical Power \& Energy Systems, vol. 8, no. 3, pp. 146157,1986

[3] S. Sastry and P. Varaiya, "Hierarchical stability and alert state steering control of interconnected power systems," IEEE Transactions on Circuits and Systems, vol. 27, no. 11, pp. 1102-1112, 1980.

[4] A. R. Bergen and D. J. Hill, "A structure preserving model for power system stability analysis," IEEE Transactions on Power Apparatus and Systems, vol. 100, no. 1, pp. 25-35, 1981.

[5] M. Ilić, "Network theoretic conditions for existence and uniqueness of steady state solutions to electric power circuits," in IEEE International Symposium on Circuits and Systems, San Diego, CA, USA, May 1992, pp. 2821-2828.
[6] A. Araposthatis, S. Sastry, and P. Varaiya, "Analysis of powerflow equation," International Journal of Electrical Power \& Energy Systems, vol. 3, no. 3, pp. 115-126, 1981.

[7] F. Wu and S. Kumagai, "Steady-state security regions of power systems," IEEE Transactions on Circuits and Systems, vol. 29, no. 11, pp. 703-711, 1982.

[8] F. F. Wu and S. Kumagai, Limits on Power Injections for Power Flow Equations to Have Secure Solutions. Electronics Research Laboratory, College of Engineering, University of California, 1980.

[9] H. Chiang and M. Baran, "On the existence and uniqueness of load flow solution for radial distribution power networks," IEEE Transactions on Circuits and Systems, vol. 37, no. 3, pp. 410-416, 1990.

[10] B. C. Lesieutre, P. W. Sauer, and M. A. Pai "Existence of solutions for the network/load equations in power systems," IEEE Transactions on Circuits and Systems I: Fundamental Theory and Applications, vol. 46, no. 8, pp. 1003-1011, 1999.

[11] S. Grijalva and P. W. Sauer, "A necessary condition for power flow Jacobian singularity based on branch complex flows," IEEE Transactions on Circuits and Systems I: Fundamental Theory and Applications, vol. 52, no. 7, pp. 1406-1413, 2005.

[12] I. Dobson, "Observations on the geometry of saddle node bifurcation and voltage collapse in electrical power systems," IEEE Transactions on Circuits and Systems I: Fundamental Theory and Applications, vol. 39 , no. 3, pp. $240-243,1992$.

[13] M. M. Hamada, M. A. A. Wahab, and N. G. A. Hemdan, "Simple and efficient method for steady-state voltage stability assessment of radial distribution systems," Electric Power Systems Research, vol. 80, no. 2 , pp. 152-160, 2010.

[14] A. Arenas, A. Díaz-Guilera, J. Kurths, Y. Moreno, and C. Zhou, "Synchronization in complex networks," Physics Reports, vol. 469, no. 3, pp. 93-153, 2008

[15] S. Boccaletti, V. Latora, Y. Moreno, M. Chavez, and D. U. Hwang, "Complex networks: Structure and dynamics," Physics Reports, vol. 424, no. 4-5, pp. 175-308, 2006.

[16] S. H. Strogatz, "Exploring complex networks," Nature, vol. 410, no. 6825, pp. 268-276, 2001.

[17] Y. Kuramoto, "Self-entrainment of a population of coupled nonlinear oscillators," in Int. Symposium on Mathematical Problems in Theoretical Physics, ser. Lecture Notes in Physics, H. Araki, Ed. Springer, 1975, vol. 39, pp. 420-422.

[18] S. H. Strogatz, "From Kuramoto to Crawford: Exploring the onset of synchronization in populations of coupled oscillators," Physica D: Nonlinear Phenomena, vol. 143, no. 1, pp. 1-20, 2000.

[19] F. Dörfler and F. Bullo, "Exploring synchronization in complex oscillator networks," in IEEE Conf. on Decision and Control, Maui, HI, USA, Dec. 2012, to appear.

[20] J. A. Acebrón, L. L. Bonilla, C. J. P. Vicente, F. Ritort, and R. Spigler, "The Kuramoto model: A simple paradigm for synchronization phenomena," Reviews of Modern Physics, vol. 77, no. 1, pp. 137-185, 2005

[21] F. Dörfler and F. Bullo, "On the critical coupling for Kuramoto oscillators," SIAM Journal on Applied Dynamical Systems, vol. 10, no. 3, pp. 1070-1099, 2011.

[22] S. H. Strogatz and R. E. Mirollo, "Phase-locking and critical phenomena in lattices of coupled nonlinear oscillators with random intrinsic frequencies," Physica D: Nonlinear Phenomena, vol. 31, no. 2, pp. 143-168, 1988.

[23] M. Verwoerd and O. Mason, "On computing the critical coupling coefficient for the Kuramoto model on a complete bipartite graph," SIAM Journal on Applied Dynamical Systems, vol. 8, no. 1, pp. 417453, 2009.

[24] F. Dörfler and F. Bullo, "Synchronization and transient stability in power networks and non-uniform Kuramoto oscillators," SIAM Journal on Control and Optimization, vol. 50, no. 3, pp. 1616-1642, 2012.

[25] A. Jadbabaie, N. Motee, and M. Barahona, "On the stability of the Kuramoto model of coupled nonlinear oscillators," in American Control Conference, Boston, MA, USA, Jun. 2004, pp. 4296-4301.

[26] T. Nishikawa, A. E. Motter, Y. C. Lai, and F. C. Hoppensteadt, "Heterogeneity in oscillator networks: Are smaller worlds easier to synchronize?" Physical Review Letters, vol. 91, no. 1, p. 14101, 2003.

[27] L. Buzna, S. Lozano, and A. Diaz-Guilera, "Synchronization in symmetric bipolar population networks," Physical Review E, vol. 80, no. 6, p. 66120, 2009.

[28] D. J. Hill and G. Chen, "Power systems as dynamic networks," in IEEE Int. Symposium on Circuits and Systems, Kos, Greece, May 2006, pp. $722-725$

[29] F. Dörfler and F. Bullo, "Kron reduction of graphs with applications to electrical networks," IEEE Transactions on Circuits and Systems I, Nov. 2011, to appear

[30] F. Dörfler, M. Chertkov, and F. Bullo, "Synchronization in complex oscillator networks and smart grids," Proceedings of the National Academy of Sciences, May 2012, to appear. 